\title{
The National Severe Storms Laboratory Historical Weather Data Archives Data Management and Web Access System
}

\author{
by Willa H. Zhu, David M. Schultz, Douglas W. Kennedy, \\ Kevin E. Kelleher, and Nancy N. Soreide
}

0 btaining historical weather data can be a frustrating experience. Finding the answers to simple questions like, "What was the temperature in Boston at 1900 UTC 23 July 1975?" can be a challenge. Many students and researchers desire historical weather data, but are unaware of where to obtain it. Popular online historical databases have limited datasets, limited capabilities, or both. The sidebar presents several sources of data available online.

The National Oceanic and Atmospheric Administration/National Severe Storms Laboratory (NOAA/NSSL) possesses several unique databases of weather information on its data-storage systems. Previously, access to these data was limited to those at NSSL understanding the General Meteorology Package (GEMPAK; www.unidata.ucar.edu/software/ gempak), an analysis, display, and product generation package for meteorological data. Upon arriving at NSSL in 1996, the second author envisioned an

AFFILIATIONS: ZHU-Joint Institute for the Study of the Atmosphere and Ocean, University of Washington, Seattle, Washington; SCHULTZ-Cooperative Institute for Mesoscale Meteorological Studies, University of Oklahoma, and NOAA/ National Severe Storms Laboratory, Norman, Oklahoma; KENNEDY-Science Applications International Corporation, and NOAA/National Severe Storms Laboratory, Norman, Oklahoma; KeLLeHER-NOAA/National Severe Storms Laboratory, Norman, Oklahoma; SOREIDE-NOAA/Pacific Marine Environmental Laboratory, Seattle, Washington

CORRESPONDING AUTHOR: Dr. David M. Schultz, NOAA/ NSSL/FRDD, Suite 4356, 120 David L. Boren Blvd., Norman, OK 73072-7326

E-mail: david.schultz@noaa.gov

DOI:10.1175/BAMS-87-12-1679

(C2006 American Meteorological Society online data portal where NSSL's historical data could be publicly viewed, manipulated, and downloaded. We are pleased to announce the creation of just such an online data portal. The first generation of this new portal is now available on the Internet at http://data. nssl.noaa.gov.

This portal is called the NSSL Historical Weather Data Archives (HWDA). The HWDA was developed by NOAA/NSSL and NOAA/Pacific Marine Environmental Laboratory (PMEL) through funding from NOAA's now-defunct Environmental Services Data and Information Management (ESDIM) program. In its current configuration, HWDA is capable of plotting surface and upper-air horizontal maps, vertical profiles, and time series from the United States and the world. Observing station locations and other information are also available. The most complete surface data come from the United States and Canada during 1973 to near real time, and the most complete upper-air data come from North America during 1948 to near real time. The purpose of this article is to describe the datasets in the HWDA, provide some information about the software, and describe its functionality.

DATASETS. Currently, two of NSSL's most popular datasets are included in the portal: surface and upper-air data. Surface data before 20 April 1998 were obtained and assembled by scientists and computer programmers at NSSL for producing climatologies of winter precipitation (Robbins and Cortinas 2002; Cortinas et al. 2004). Two surface datasets from the NOAA/National Climatic Data Center (NCDC) were quality controlled and merged into a single format, as described in Robbins and Cortinas (2002). The first dataset is TD-3280, the U.S. surface airway hourly observations from NCDC. The second dataset is 
- National Climatic Data Center. Numerous online datasets for the United States and the world: precipitation, Weather Surveillance Radar-1988 Doppler (WSR-88D) radar mosaics, surface data, climatic normals, etc. Many datasets are free to the public. No ability to plot some data. Many datasets are not available in real time. http://www.ncdc.noaa.gov

- University of Wyoming upper-air soundings. Plots vertical profiles and lists worldwide upper-air data in a variety of formats from 1973 to present. Excellent real-time source of upper-air data. No ability to plot constant-pressure maps. http://weather.uwyo. edu/upperair/sounding.html
- Plymouth State Weather Center product generator. Plots surface maps (data and contours) and time series (1998-yesterday), upper-air data (1957-yesterday), satellite (1996-yesterday), and WSR-88D radar maps (1999-yesterday). http://vortex.plymouth.edu/u-make.html

- National Severe Storms Laboratory Historical Weather Data Archives. Plots vertical profiles, draws constant-pressure maps, and lists upper-air data from the United States and Canada for 1946-2003 and worldwide 2000-03. Plots surface maps and lists surface data from the United States and Canada for 1973 to 2003 , with some data from as early as 1933 and worldwide 1998-2003. Datasets are only available in near real time. http://data.nssl.noaa.gov
DATSAV2, a surface dataset from the U.S. Air Force Combat Climatology Center. Although data exist in this dataset as early as 1933, the earliest data are sparse and primarily from military bases. The most complete data for constructing surface maps are found for 1973 to near real time. Hourly data are available, minus the off-hour observations. Since 20 April 1998, the surface data come from the operational datastream over the Global Telecommunications System archived by NSSL and the NOAA/National Weather Service/Storm Prediction Center (SPC). Although some worldwide data exist in the dataset before 1998, the majority of the available worldwide data occur after this time.

Upper-air data before 2000 come from the North American Radiosonde Database created by the NOAA/Forecast Systems Laboratory (FSL), now the Global Systems Division of the Earth System Research Laboratory. More information on this dataset can be found in Schwartz and Govett (1992) and at the FSL/NCDC Radiosonde Data Archive Web site (http://raob.fsl.noaa.gov/Raob_Software.html). Since 1 January 2000, the upper-air data come from the operational datastream over the Global Telecommunications System archived by NSSL and SPC. At this point in the dataset, the availability of worldwide upper-air data increases.

DATA MANAGEMENT. Both surface and upperair datasets are maintained on an NSSL data server computer. The data are stored as one GEMPAK file per day, where surface datasets contain hourly data and upper-air datasets contain data at least every 12 hours.
The metadata of the archives are managed using mySQL database and are available for both the surface and upper-air data. MySQL is an open-source database management system. Data listings of station information contain latitude, longitude, elevation, time range of data availability, station identifier, and World Meteorological Organization (WMO) number, all derived from GEMPAK station tables. The most recent data in the dataset also contain these metadata.

ONLINE DATA ACCESS. The NSSL HWDA (Fig. 1) was developed to provide convenient access to these historical surface and upper-air weather data. The NSSL HWDA Web site offers interactive data access selection features. The world is divided into

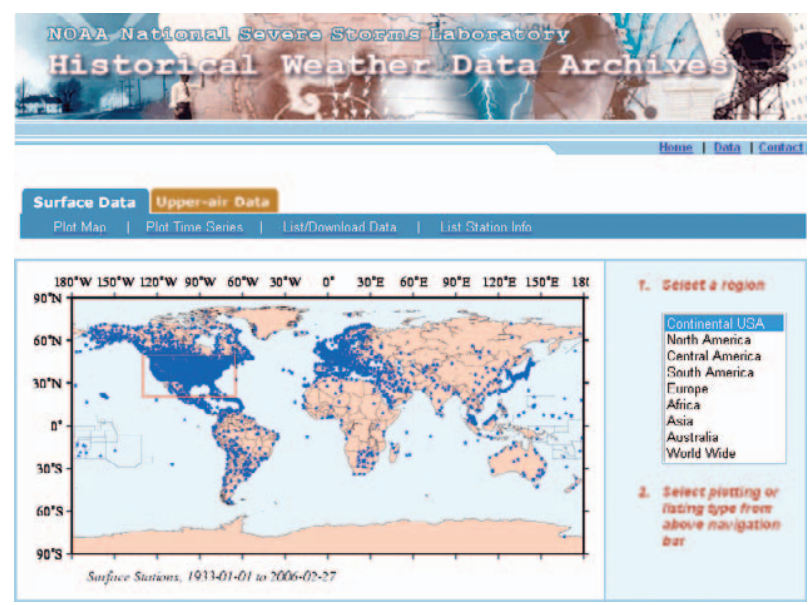

FIG. I. The NSSL Historical Weather Data Archives Web site (http://data.nssl.noaa.gov). 


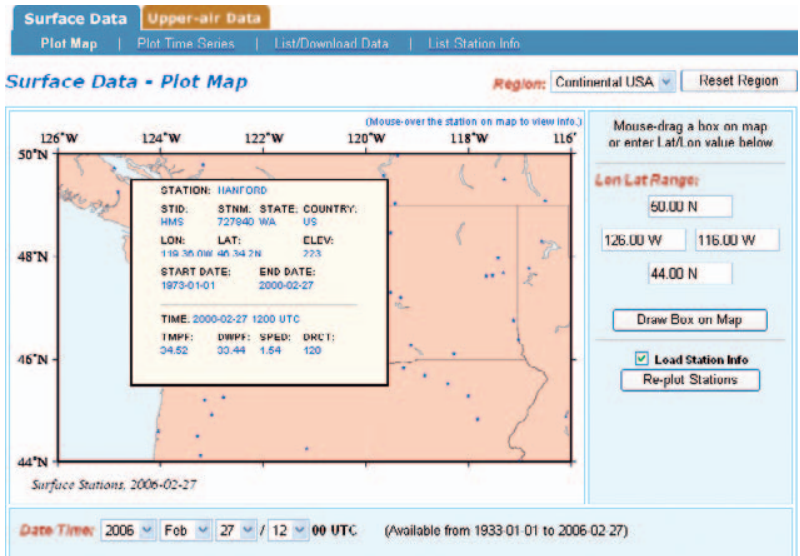

FIG. 2. Moving the mouse over a station reveals a listing of information about the station.

several major regions (e.g., continental United States, Europe, Africa). Users select a region of interest and then can zoom in to a smaller domain by dragging a "rubberband box" over the map. By selecting the button, "Load station info," and reloading the page, users can view station information when the mouse is positioned over a station (Fig. 2). Station data can also be listed for all stations within the plotted domain.

GEMPAK serves as a backend plotting and listing tool. The NSSL HWDA Web site initiates GEMPAK programs through scripts to make user-defined plots and lists of data. Another piece of graphic software, Generic Mapping Tools (GMT; http://gmt.soest.hawaii.edu), generates geographic station location maps to provide Web interactive station/area selection.

Dynamic HTML (DHTML) technologies have been implemented in the NSSL HWDA Web interface system. These include JavaScript, dynamic styles, cascading style sheets, and other technologies to dynamically change HTML displays. In particular, the DHTML technologies are used to show and hide an HTML layer dynamically. When users move the mouse toward or away from the station location on a map, the layer containing information about that station is shown or hidden, respectively. The station information is loaded dynamically by accessing the backend database. DHTML is also implemented when dragging a rubberband box over a map to select an area. Four HTML layers containing each side of the box are shown as the mouse is dragged over the map. The corresponding geographic location is converted into pixel location on the map so the layers are shown in the correct location and length. DHTML is a modern replacement for Java applets as an improved, fast-response, interactive user interface for the Web.

PHP (a server-side scripting language) is used to communicate with the backend database, initiate graphic tools and scripts, and generate the desired page dynamically. This language also provides reusable components for the Web site. The HWDA has been tested successfully on Internet Explorer and Firefox on PCs and using Netscape on Macintosh. Macintosh's Safari and Firefox browsers are not fully compatible with the HWDA.

CAPABILITIES OF THE HWDA. The current configuration of the HWDA delivers surface and upper-air data to the user through horizontal maps, vertical profiles on skew $T-\log p$ charts, time series, and ASCII data listings (Fig. 3). Users arriving at the HWDA to view or download data are first asked to select a region of the world from a drop-down menu of choices. Users can later subset the map for smaller domains, focusing just on a state or region by drawing a box around the area of interest. Because the amount of plotted data is filtered for legibility for larger domains, plotting smaller domains is useful for seeing data from a greater number of stations, especially at the surface. Users then select surface or upper-air data and their choice of plot type or data listing. For surface data, users can create a surface map with plotted station models, time series, or data listing. For upper-air data, users can create upper-level maps (in height, pressure, or isentropic coordinates), time series, vertical profiles on skew $T-\log p$ charts, or data listings. Because the surface and upper-air data are stored hourly in 24 -h data files, time series are limited to 0000 to 2300 UTC. Whereas 24 -h time series of surface data may be useful to many, such time series will not be very useful for twice-a-day upper-air data. We hope future versions of the software may be able to accommodate longer time series.

One advantage of the HWDA is that users can make a minimum of selections and get plots and data listings quickly, yet more sophisticated users familiar with GEMPAK commands have the ability to exert some control over the manner in which the plots are presented, altering the standard station model, for instance, or requesting nonstandard fields, like equivalent potential temperature. Links to those GEMPAK commands are provided on the Web page.

CONCLUSIONS. The NSSL Historical Weather Data Archives interactive Web access system pro- 


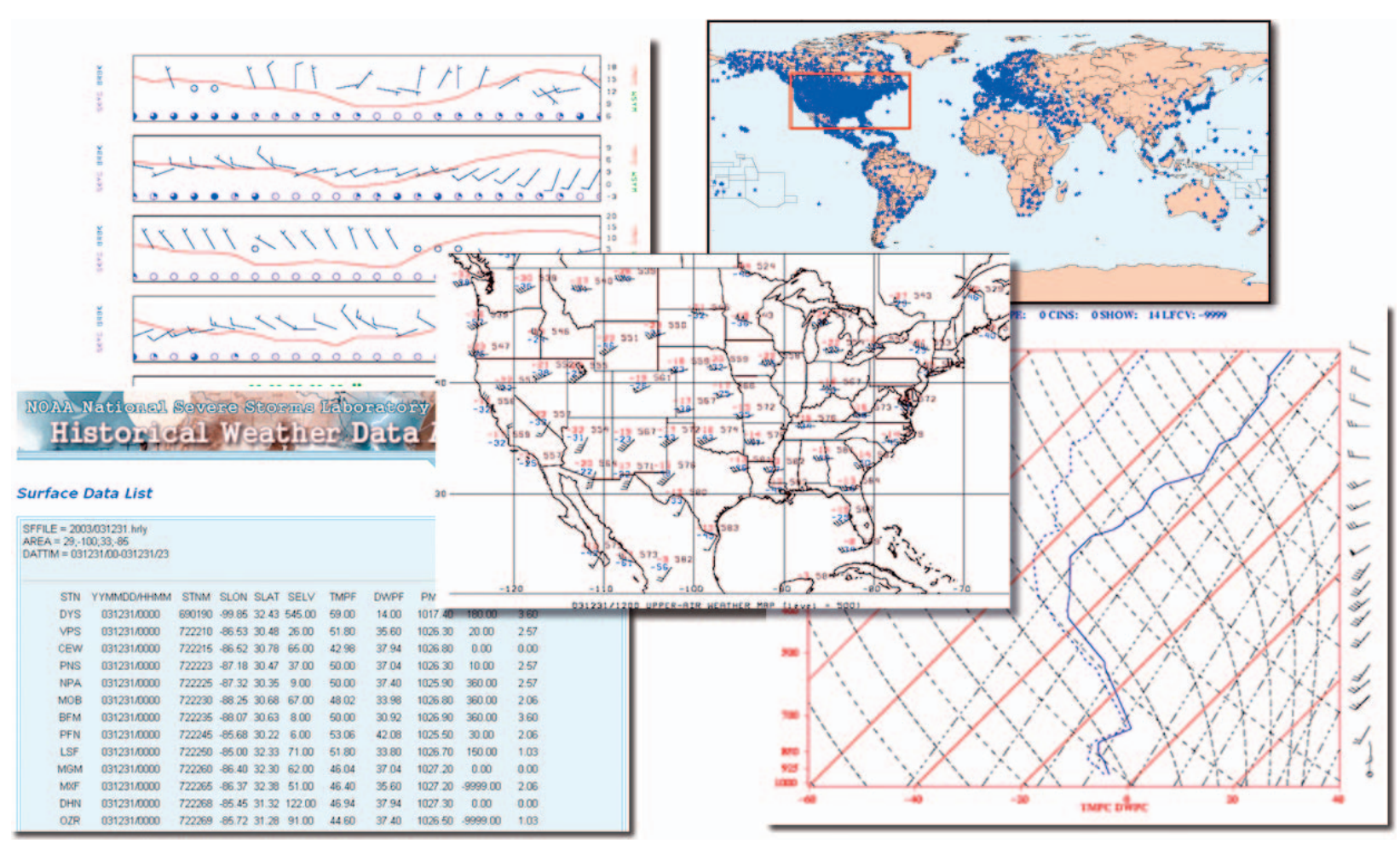

FIG. 3. Examples of output from NSSL HWDA (from left to right and clockwise): listing of surface data, time series, upper-air constant-pressure map, selection window for choosing region of world, and skew T-log $p$ chart.

vides an easy and convenient way for scientists and other users to access historical weather data. The system is customized for GEMPAK, but can be extended to support other data formats with different applications and tools.

Although this first generation of the HWDA delivers data from the surface and upper-air datasets archived at NSSL, we have plans for future versions. A more complete surface dataset for the United States prior to 1973 is desired. Other datasets at NSSL could also be added to the HWDA, particularly data from numerous NSSL field programs and the National Lightning Detection Network (limited distribution of these data is required because of their commercial nature). A third improvement would be the ability to segment large chunks of data for creating composite maps (e.g., "give me all the dates where the temperature exceeded $100^{\circ} \mathrm{F}$ at Albany, New York").

The NSSL HWDA is now available on the Internet, free to all. The data and plots produced by the HWDA may be useful in synoptic laboratories, classroom exercises, research projects, and in publications. Properly crediting our images and data in publications will assist us in being able to continue improving this Web site. For example, "Image provided by the National Severe Storms Laboratory Historical Weather Data Archives, Norman, Oklahoma, from their Web site at http://data.nssl.noaa.gov." We welcome feedback on this data portal; please send your comments to nssl.data@noaa.gov.

ACKNOWLEDGMENTS. The NSSL Historical Weather Data Archives Web page was developed by NOAA/NSSL and NOAA/PMEL under funding from NOAA's Environmental Services Data and Information Management program. This publication is partially funded by the Joint Institute for the Study of the Atmosphere and Ocean under NOAA Cooperative Agreement NA17RJ1232, Contribution 2002, and is NOAA/PMEL Contribution 2883. Partial funding for Schultz was provided by NOAA/Office of Oceanic and Atmospheric Research under NOAA-University of Oklahoma Cooperative Agreement NA17RJ1227, U.S. Department of Commerce. Special thanks to Joan O'Bannon for designing the banner graphics for the HWDA, Neal Lott for providing the surface dataset, three anonymous reviewers for comments that improved the manuscript and Web page, and John Cortinas, Steve Fletcher, and Chris Robbins for creating the merged surface dataset. 


\section{FOR FURTHER READING}

Cortinas, J. V., Jr., B. C. Bernstein, C. C. Robbins, and J. W. Strapp, 2004: An analysis of freezing rain, freezing drizzle, and ice pellets across the United States and Canada: 1976-90. Wea. Forecasting, 19, 377-390.

desJardins, M. L., K. F. Brill, and S. S. Schotz, 1991: Use of GEMPAK on Unix workstations. Preprints, Seventh Int. Conf. on Interactive Information and Processing Systems for Meteorology, Oceanography, and Hydrology, New Orleans, LA, Amer. Meteor. Soc., 449-453.

Koch, S. E., M. desJardins, and P. J. Kocin, 1983: An interactive Barnes objective map analysis scheme for use with satellite and conventional data. J. Climate Appl. Meteor., 22, 1487-1503.

Robbins, C. C., and J. V. Cortinas Jr., 2002: Local and synoptic environments associated with freezing rain in the contiguous United States. Wea. Forecasting, $17,47-65$.

Schwartz, B., and M. Govett, 1992: A hydrostatically consistent North American radiosonde data base at the Forecast Systems Laboratory, 1946-present. NOAA Tech. Memo. ERL FSL-4, 81 pp. [Available from NOAA/ERL/FSL, 325 Broadway, Boulder, CO 80303; CD-ROM available from National Climatic Data Center, Federal Building, 151 Patton Avenue, Asheville, NC 28801-5001.] 